\title{
Estimación de la evapotranspiración de referencia mediante atmómetro con fines de calendarización de riego
}

\author{
Estimation of reference evapotranspiration using \\ an atmometer for irrigation scheduling \\ Cándido Mendoza-Pérez ${ }^{*}$, Waldo Ojeda-Bustamante2, \\ Ernesto Sifuentes-Ibarra ${ }^{3}$, Abel Quevedo-Nolasco', Héctor Flores-Magdaleno ${ }^{1}$, \\ Carlos Ramírez-Ayala ${ }^{1}$, Roberto Ascencio-Hernández ${ }^{1}$
}

\section{RESUMEN}

En zonas áridas y semiáridas la evapotranspiración representa gran pérdida de agua hacia la atmósfera. Por eso es necesario entender y cuantificar este proceso. El trabajo se realizó en el Campo Experimental Valle del Fuerte durante el ciclo agrícola otoño-invierno 2012-13. Se utilizó una estación meteorológica para medir las variables climáticas y el atmómetro para calcular la ETo. Se obtuvo el cuadrado medio del error (MSE), raíz del cuadrado medio del error (REMC) y la regresión lineal para evaluar estadísticamente el desempeño del atmómetro. La estimación de ETo fue de 1495 y $1455 \mathrm{~mm}$ para el atmómetro y la estación meteorológica, respectivamente. El coeficiente de regresión lineal fue de $\mathrm{R}^{2}=87 \%$ y el MSE y REMC fueron de 0,71 y 0,84 mm. El rendimiento fue de 2,66 y 2,64 th a ${ }^{-1}$ para el atmómetro e IrriModel, con productividad del agua de $0,71 \mathrm{y} 0,68 \mathrm{~kg} \mathrm{~m}^{-3}$. El análisis de regresión lineal del atmómetro presenta alto nivel de confianza para estimar la ETo. Con manejo y mantenimiento adecuado el atmómetro es una alternativa para calcular la ETo con fines de calendarización del riego en cultivos.

Palabras clave: Phaseolus vulgaris L, estación meteorológica automática, riego de precisión, rendimiento.

\begin{abstract}
In arid and semi-arid zones, evapotranspiration represents a great loss of water into the atmosphere, which is why it is necessary to understand and quantify this process. The work was carried out in the Valle del Fuerte Experimental Field during the autumn-winter 2012-13 agricultural cycle. A meteorological station was used to measure the climatic variables and the atmometer to measure ETo. The mean square of the error (MSE), mean square root of the error (CTMR) and the linear regression were obtained to statistically evaluate the performance of the atmometer. The estimate of ETo were 1495 and $1455 \mathrm{~mm}$ for the atmometer and the weather station. The linear regression coefficient was $R^{2}=87 \%$, the MSE and CTMR were 0.71 and $0.84 \mathrm{~mm}$. The yield was 2.66 and 2.64 th $a^{-1}$ for the atmometer and IrriModel, with water productivity of 0.71 and $0.68 \mathrm{~kg} \mathrm{~m}^{-3}$. The linear regression analysis of the atmometer presents a high level of confidence to estimate ETo. The atmometer with proper management and maintenance is an alternative to estimate the ETo for the scheduling of irrigation in crops.
\end{abstract}

Keywords: Phaseolus vulgaris L, automatic weather station, precision irrigation, yield.

\section{Introducción}

La evapotranspiración es una de las principales pérdidas de agua en regiones semiáridas. Por este motivo su estimación es importante para hacer los balances hídricos en suelos agrícolas y con vegetación natural (Domingo et al., 1999). La disponibilidad de los recursos hídricos para la agricultura es cada vez menor, por el crecimiento de la población, la competencia por diferentes sectores en el uso del agua, la ocurrencia de sequía y degradación de calidad del agua. Es por ello que la medición de la evapotranspiración en la agricultura es una variable importante (García et al., 2004).

\footnotetext{
Departamento de Hidrociencias del Colegio de Postgraduados. Montecillo, Estado de México, México.

Instituto Mexicano de Tecnología del Agua. Colonia Progreso. Jiutepec, Morelos, México.

INIFAP-CIRNO-Campo Experimental. Juan José Ríos. Guasave, Sinaloa, México.

* Autor por correspondencia: mendoza.candido@colpos.mx.
}

Fecha de Recepción: 11 Junio, 2019.

Fecha de Aceptación: 11 Septiembre, 2019. 
Su estimación es difícil a través de métodos indirectos sin requerir de equipos costosos disponibles (Melesse et al., 2007). Experimentalmente, la evapotranspiración se mide con lisímetros de pesada, técnicas de Eddy covariance o relación de Bowen. Sin embargo, estos métodos son limitados, ya que proporcionan valores puntuales de evapotranspiración para un lugar específico y no la evapotranspiración a una escala regional. En cambio los atmómetros suministran datos a escala regional (Melesse et al., 2007; Gordillo et al., 2014). Actualmente existen métodos para estimar a escala local las necesidades hídricas de un cultivo, con base en dos principios fundamentales: balance de energía o balance hídrico (Melesse et al., 2007).

La metodología más usada para medirla se basa en el conocimiento de dos factores: I) el coeficiente de cultivo $(K c)$ que depende de la especie y la etapa del cultivo, y II) evapotranspiración de referencia (ETo). Allen et al. (1998) han documentado ampliamente el uso de la ecuación ETc $=K c * E T o$ para estimar la evapotranspiración de los cultivos.

Por su simplicidad, bajo costo y registro de datos, el uso del tanque evaporímetro tipo A ha sido una alternativa para calcular la ETo en varias regiones agrícolas. Como fue reportado por Fernández et al. (2003), el uso de tanques evaporímetros requiere de una calibración previa, además del espacio, mantenimiento y supervisión. Ante estas desventajas, el uso comercial de tanques evaporímetros con fines de calendarización de riego es limitado (Knox et al., 2011). Existe un interés por el uso de atmómetros para estimar la ETo por ser una alternativa viable (Knox et al., 2011), pero se requiere su calibración y validación local. No existe evidencia del empleo de atmómetros con fines de calendarización del riego para las condiciones de las zonas agrícolas localizadas en las llanuras costeras secas de México.

Conocer la evapotranspiración de referencia y el requerimiento hídrico de los cultivos permite una programación científica del riego, oportuna y eficiente, $y$, en consecuencia, se incrementa la eficiencia de aplicación del agua y fertilizantes para obtener semillas de mejor calidad. El objetivo de este trabajo fue calibrar la confiabilidad del atmómetro con el fin de estimar la evapotranspiración de referencia (ETo) para calendarización de riego. Posteriormente se usaron los datos para la programación de riego en dos parcelas experimentales (Atmómetro e IrriModel) mediante riego por goteo en frijol. Además se midió el rendimiento y la productividad del agua.

\section{Materiales y métodos}

El trabajo se realizó en el Campo Experimental Valle del Fuerte (CEVAF) del Instituto Nacional de Investigaciones Forestales, Agrícolas y Pecuarias (INIFAP), localizado en la zona árida del Norte de Sinaloa, México $\left(25,76^{\circ}\right.$ latitud Norte y $108.86^{\circ}$ longitud Oeste a $20 \mathrm{~m}$ de altura). El atmómetro se instaló a $1.2 \mathrm{~m}$ de altura con respecto al suelo recomendado por Vázquez-Méndez et al., 2011, junto a una estación meteorológica automática ubicada a $20 \mathrm{~m}$ de la parcela experimental. El experimento se estableció en un suelo de textura arcillosa, humedad volumétrica aprovechable de $0,155 \mathrm{~cm}^{3} \mathrm{~cm}^{-3}$, densidad aparente de $1,2 \mathrm{~g} \mathrm{~cm}^{-3} \mathrm{y}$ contenido de materia orgánica de $0,78 \%$. La parcela es parte del Distrito de Riego 075, Río Fuerte, Sinaloa (CONAGUA, 2015). La precipitación anual acumulada en la zona de estudio es de $350 \mathrm{~mm}$ y el $70 \%$ se concentra desde julio hasta septiembre y el resto se distribuye en los meses de febrero a mayo. Los valores anuales de la evapotranspiración de referencia acumulada varían de 1600 a $1700 \mathrm{~mm}$, lo cual excede la precipitación de todo el año. Por ello se requiere de riego para asegurar rendimientos comerciales de los cultivos de la zona (MendozaPérez et al., 2016).

La temperatura media anual de la zona se encuentra entre 24 y $26^{\circ} \mathrm{C}$. Junio es el mes más caliente y enero el más frío. La temperatura mínima es de alrededor de $10,5^{\circ} \mathrm{C}$. En los meses de mayo a septiembre se tienen temperaturas máximas cercanas a $45^{\circ} \mathrm{C}$, lo que genera estrés térmico en la mayoría de los cultivos agrícolas (MendozaPérez et al., 2016).

\section{Descripción del atmómetro}

El atmómetro consiste en un plato poroso de cerámica conectado internamente con una manguera a un reservorio cilíndrico de agua destilada. Este aparato simula la resistencia de difusión del vapor de agua de la superficie porosa evaporante. Usualmente se coloca una membrana de politetrafluoroetileno sobre el plato para prevenir la entrada de agua a través de este (Chen y Robinson, 2009). Además tiene dos alambres rígidos de acero inoxidable 
de dos pulgadas en la parte superior para evitar que las aves se posen sobre el medidor (Figura 1).

Este modelo dispone de un depósito de agua de $300 \mathrm{~mm}$, fabricado en PVC blanco para reflejar la radiación solar y evitar la transmisión de calor al agua que está almacenada en su interior.

\section{Toma de datos}

Se utilizó un atmómetro (ETgage, Modelo A comercializado por la ETgage Company (Loveland, Colorado, EE UU). Se instaló a $1.2 \mathrm{~m}$ de altura, y la cantidad de agua evaporada en las 24 horas previas se midió manualmente a las 8:00 am, a través de un tubo de cristal con una escala graduada montado en la parte exterior del equipo (Figura 1). La lámina de agua evaporada se estimó por el cambio en dos niveles de agua de dos lecturas consecutivas. El periodo en la toma de datos fue desde noviembre 2012 hasta agosto de 2013.

El resto de las variables meteorológicas se obtuvieron de una estación meteorológica automática, que opera el Instituto Nacional de Investigaciones Forestales, Agrícolas y Pecuarias (INIFAP) y que está instalada a $20 \mathrm{~m} \mathrm{del}$ experimento. Se miden diariamente las siguientes variables: temperatura máxima y mínima, humedad relativa, precipitación pluvial, radiación solar, dirección y velocidad del viento.

\section{Descripción de la estación meteorológica automática}

La estación meteorológica automática es una estructura o dispositivo con sensores electrónicos que responden a estímulos de las variables meteorológicas, con capacidad de colectarlas y registrarlas en forma automática y en tiempo real. Genera datos en intervalos de 15 minutos de las variables meteorológicas. Esta información es enviada vía satélite en intervalos de 1 ó 3 horas por estación (Referencia OMM 182). El horario que utiliza para el registro es el TUC ó UTC (Tiempo Universal Coordinado) y por esta razón deberá tener en consideración ese factor para la correcta interpretación de los datos.

\section{Estimación del error}

Los datos de ETo del atmómetro fueron comparados con los datos de ETo obtenidos de la estación meteorológica automática utilizando el coeficiente de regresión lineal. El Error Cuadrático Medio (EMA) y la Raíz del Cuadrado Medio del Error (RMSE) se calcularon con la Ecuación 1 y 2.

$\mathrm{EMA}=\frac{1}{\mathrm{n}} \sum_{\mathrm{i}=1}^{\mathrm{n}}(\text { ETo estacion, } \mathrm{i}-\text { ETo atm }, \mathrm{i})^{2}$

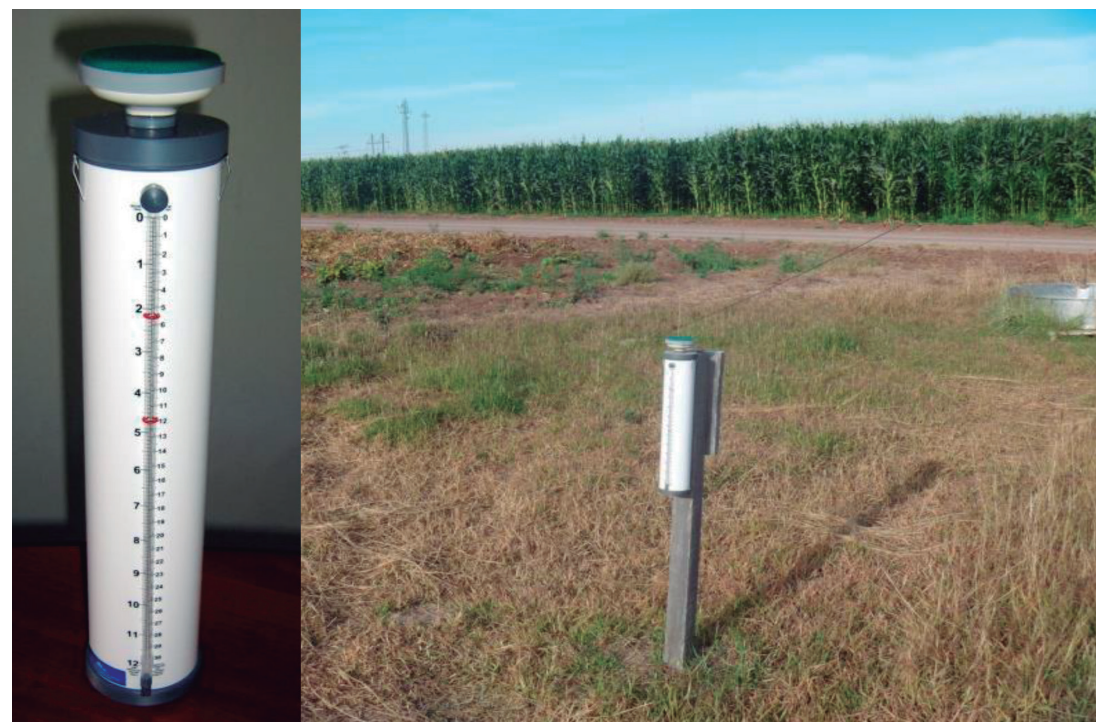

Figura 1. Atmómetro instalado en la zona de estudio. 
$\mathrm{RMSE}=\sqrt{\frac{1}{\mathrm{n}}} \sum_{\mathrm{i}=1}^{\mathrm{n}}(\text { ETo estacion, } \mathrm{i}-\text { ETo } \mathrm{atm}, \mathrm{i})^{2}$

Donde: $E T o_{\text {estación }}$, i y ETo atmómetro $_{\text {i }}$ i= valores del modelo y datos observados respectivamente, para cada día i, y n es el número total de días. EMA y RMSE están en unidades (mm), y son indicadores agregados del desempeño del modelo, mientras que los estadísticos de regresión (pendiente, intercepto, $\mathrm{R}^{2}$ ) son indicadores de predicción de los modelos de la ETo en una escala de tiempo (Douglas et al., 2009; Gavilán y Castillo-Llanque, 2009).

\section{Calendarización del riego con los valores de ETo del atmómetro}

Para utilizar los datos de ETo obtenidos del atmómetro en la calendarización del riego, se establecieron dos parcelas experimentales de cultivo de frijol con fecha de siembra de 12 de diciembre del 2012, de la variedad Noroeste de ciclo intermedio. Además se aplicó una fertilización base previo a la siembra de $100 \mathrm{~kg} \mathrm{ha}^{-1}$ de MAP (fosfato monopotásico) y $200 \mathrm{~kg} \mathrm{ha}^{-1}$ de urea con densidad de siembra de 10,000 semillas por hectárea, bajo sistema de riego por goteo.

Parcela 1 (Atmómetro): la programación del riego se realizó con los datos de ETo del atmómetro y el coeficiente de cultivo con valores de 0,$4 ; 1,10$ y 0,5 para kc (inicial, media y final). El cálculo de la evapotranspiración de cultivo se hizo con la ecuación 3.

Cálculo de la evapotranspiración de cultivo.

$$
E T C=E T o * \mathrm{kc}
$$

Donde: ETc es evapotranspiración de cultivo $(\mathrm{mm}), E T o=$ evapotranspiración de referencia (mm), $K c=$ coeficiente de cultivo.

Cálculo de lámina de riego.

$$
\mathrm{Lr}=\mathrm{Etc} / \mathrm{EA}
$$

Donde: $\mathrm{Lr}=$ lámina de riego $(\mathrm{mm}), E T c=$ evapotranspiración de cultivo $(\mathrm{mm}), \mathrm{EA}=$ eficiencia de aplicación (\%).

Experimento 2 (IrriModel): la programación del riego se realizó con el software (IrriModel) donde está integrada la ecuación Penman-Monteith para estimar la ETo de la estación y el resto de las variables meteorológicas se obtuvieron de la estación meteorológica automatizada para calcular el calendario de los riegos en tiempo real.

El software IrriModel opera a través de internet y pronostica los riegos en tiempo real, en función de fecha de siembra, tipo de suelo, tipo de cultivo, etapa fenológica del cultivo, humedad del suelo y las variables climáticas para estimar la programación de riego en los cultivos. El software mide diariamente los parámetros de calendarización en función de los (GDA) Grados Día Acumulados (Ojeda et al., 2004).

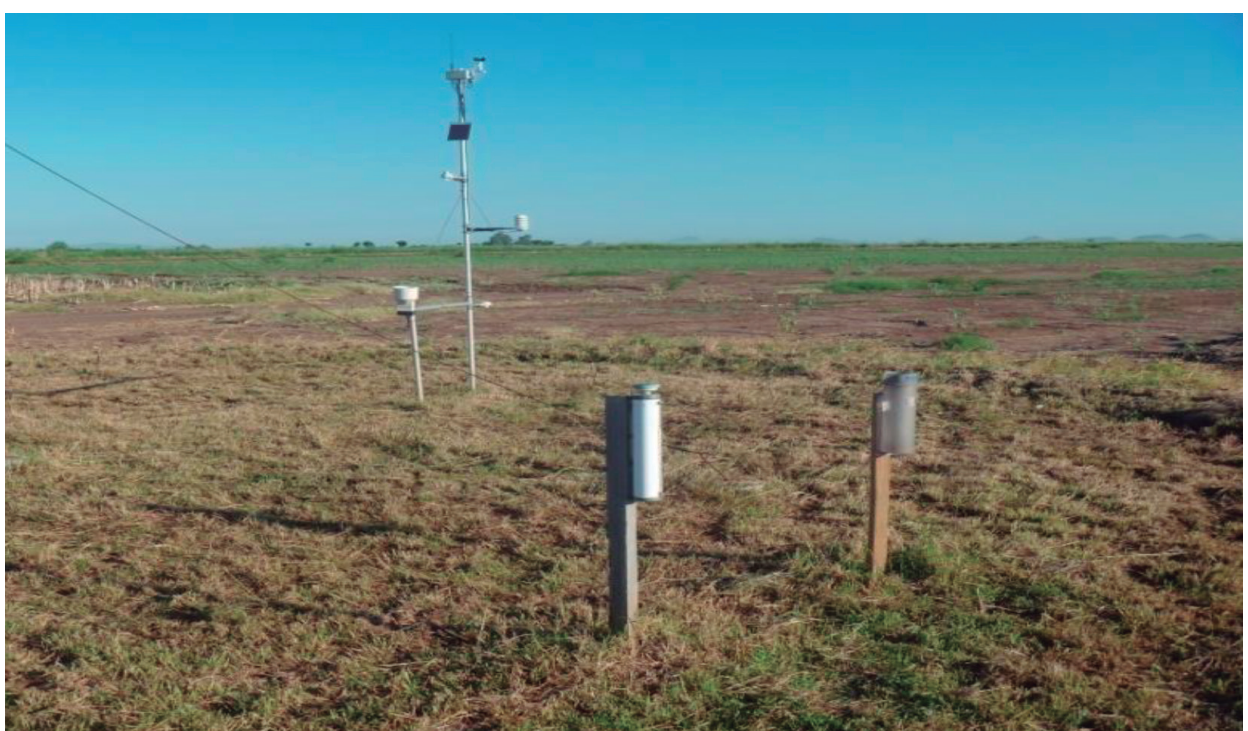

Figura 2. El atmómetro se instaló junto a una estación meteorológica automática. 


\section{Error residual}

Este error se obtuvo de la diferencia entre los valores de ETo de la estación meteorológica automática y los valores de ETo del atmómetro.

\section{Productividad del agua}

Se estimó también la productividad del agua (PA) y el Rendimiento (R) de las parcelas experimentales. La primera indica la relación de la producción total obtenida ( $\mathrm{RC}, \mathrm{kg}$ ) con respecto al volumen de agua total aplicada (VA, $\mathrm{m}^{3}$ ) (Bessembinder et al., 2005) y la segunda la producción obtenida en $\mathrm{kg} \mathrm{ha}^{-1}$.

\section{Resultados y discusión}

\section{Calibración y comparación de ETo del atmómetro con la estación meteorológica automática}

Los datos de ETo obtenidos de la estación meteorológica automática comparados con los del atmómetro (Figura 3. a) presentan gráficamente el comportamiento de la ETo a nivel diario desde el momento en que se inició la toma hasta el último día. Esto indica que el atmómetro es una herramienta robusta y confiable para la calendarización de riego en cultivos de la zona de estudio. Los datos fueron colectados a partir del 8 de noviembre de 2012 hasta el 15 de agosto de 2013. Hadi y Farah (2018) reportaron comportamientos similares al comparar la ETo diaria medida del atmómetro con la ETo diaria estimada con la ecuación de Penman-Monteith.

El valor más alto de ETo del atmómetro fue de $10,5 \mathrm{~mm}$ y $9 \mathrm{~mm}$ el de la estación meteorológica. Estos valores se registraron a finales de junio de 2013. El valor más bajo de ETo del atmómetro fue de 0,8 y $0,5 \mathrm{~mm}$ para la estación meteorológica, y se registraron el 31 de diciembre de 2012.

Se comparó también la variación de la velocidad del viento con la ETo del atmómetro, y se aprecia que a medida que aumenta la velocidad del viento se incrementa la ETo debido a la mayor pérdida de vapor de agua por la componente aerodinámica. Asimismo se observa que en los meses de marzo a julio la velocidad del viento y la ETo son elevadas. Esto coincide con los meses más calientes de la zona (Figura 3.b).

\section{Humedad relativa con la ETo del atmómetro}

Al comparar la humedad relativa (HR) con la ETo del atmómetro, se evidenció que a medida que se incrementa la HR en el ambiente disminuye el valor de la ETo. También se determina que en los meses de diciembre y enero la humedad relativa se encuentra alrededor del $80 \%$ que coincide con

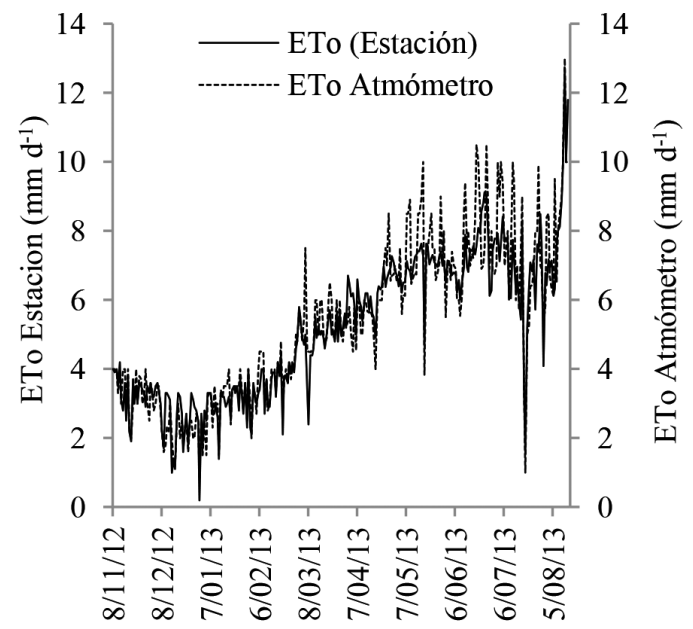

Fechas de lecturas

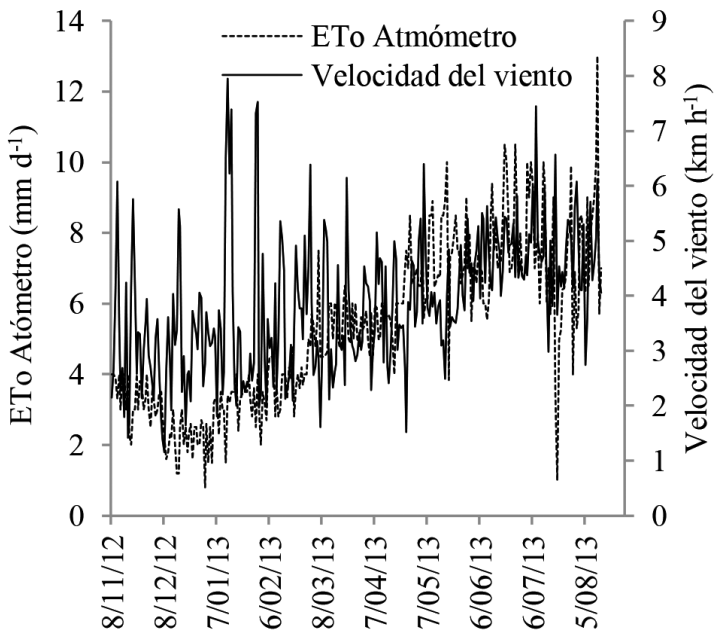

Fecha de lecturas

Figura 3. Relación entre la ETo medida con el atmómetro y la estación meteorológica automática a) y relación entre la ETo del atmómetro y la velocidad del viento b). 
la temporada de frío de la zona y por lo tanto la ETo disminuye. A partir de marzo desciende la HR y incrementa la evapotranspiración de referencia (Figura 4.a). Se observa que a medida que aumenta la temperatura del ambiente también se incrementa la evapotranspiración de referencia (Figura 4.b).

\section{Coeficiente de regresión lineal}

Se puede apreciar una regresión lineal entre ambos métodos de estimación de la evapotranspiración de referencia. La dispersión de los puntos tiene un patrón definido. El análisis de regresión lineal es positivo, lo cual indica que las variables son dependientes y se pueden explicar mutuamente con una confianza del 87\% (Figura 5). Hadi y Farah (2018) obtuvieron una regresión lineal de $84 \%$ al correlacionar ETo diario medido (atmómetro) y ETo estimado (Penman-Monteith) bajo condiciones de invernadero. Además, Gavilán y Castillo-Llanque (2009) reportaron que los atmómetros en condiciones exteriores sobreestiman la ETo PM (Penman-Monteith) en períodos secos y también en días con altas temperaturas máximas $\left(>35^{\circ} \mathrm{C}\right)$ y altas velocidades del viento $(<2 \mathrm{~m}=\mathrm{s})$.

\section{Desempeño de los modelos}

La evapotranspiración de referencia acumulada fue de $1455 \mathrm{~mm}$ con datos estimados de la estación meteorológica automática y $1495 \mathrm{~mm}$ con el atmómetro, con una diferencia de $40 \mathrm{~mm}$. El Cuadrado Medio del Error (MSE) y la Raíz del Cuadrado Medio del Error (RMSE) fueron de 0,71 y 0,84 . El margen de error entre ambos métodos fue menor de $1 \mathrm{~mm}$ (Tabla 1). Los resultados obtenidos en este trabajo son similares a los datos reportados por Vázquez-Méndez, et al. (2011) con valores de 0,98 de (EMA) y 1,27 (REMC) a nivel diario.

\section{Error residual del atmómetro}

El error residual se refiere al error que se comete al momento de tomar las lecturas del atmómetro. El error más común es no establecer un horario predeterminado para realizar la toma de las lecturas. Otro problema son las condiciones de acceso al lugar, y esto es muy común en temporadas de lluvias en la zona. A partir de 173 días (23 de abril 2013) hasta 271 días (04 de agosto) se observa de manera notoria la variación del error que, además, coincide con meses lluviosos en la zona de estudio (Figura 6).

\section{Calendarización de riego en cultivo de frijol}

La Tabla 2 presenta el resumen de los riegos aplicados en el cultivo de frijol, con datos de ETo del atmómetro. Se aplicó un total de 16 riegos incluyendo el de germinación con una lámina bruta de $37,55 \mathrm{~cm}$ para la parcela del atmómetro y $38,87 \mathrm{~cm}$ para la parcela de IrriModel, con una diferencia de $1,23 \mathrm{~cm}$.

\section{Rendimiento de las parcelas experimentales}

El rendimiento obtenido fue de 2,66 th $\mathrm{a}^{-1}$ en la parcela de atmómetro y 2,64 th $\mathrm{a}^{-1}$ en la parcela

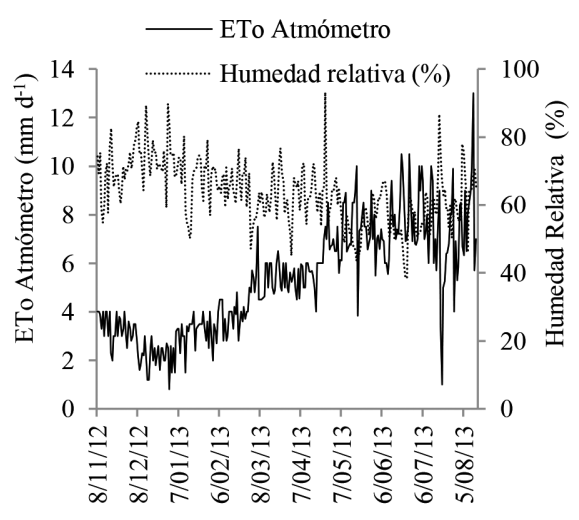

Fecha de lecturas

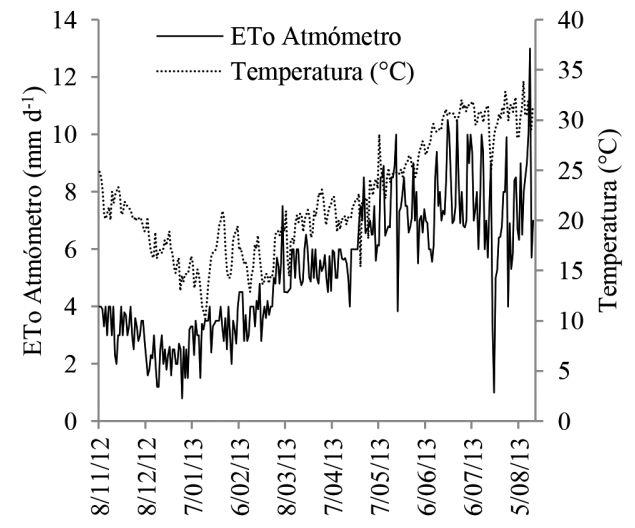

Fechas de lecturas

Figura 4. Relación temporal de la ETo del atmómetro y la humedad relativa (a) y ETo del atmómetro y la temperatura (b). 


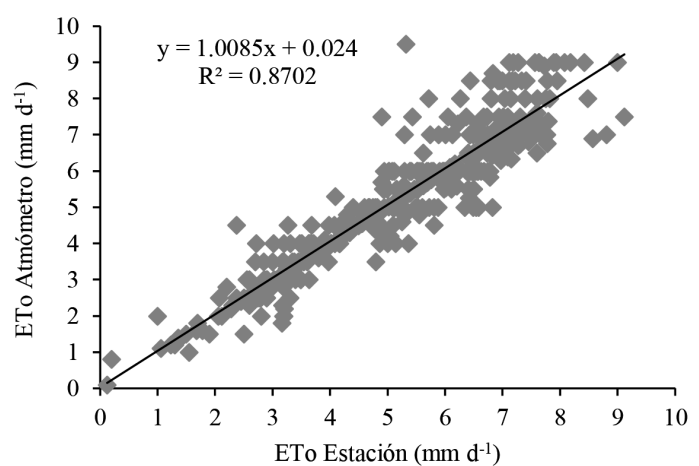

Figura 5. Coeficiente de regresión lineal de ETo entre ambos métodos.

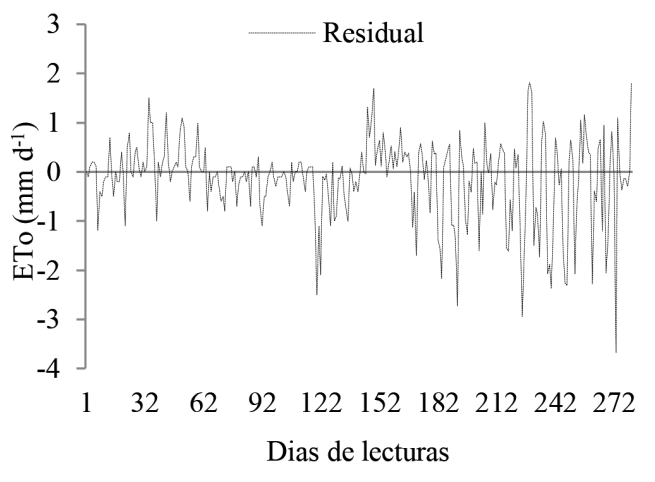

Figura 6. Error realizado en la toma de datos.

Tabla 1. Estadístico del Cuadrado Medio del Error y la Raíz del Cuadrado Medio del Error a nivel diario.

\begin{tabular}{lcccc}
\hline Método & $\begin{array}{c}\mathrm{N}^{\mathbf{0}} \\
\text { datos }\end{array}$ & $\begin{array}{c}\text { ETo } \\
\text { (Acumulado) } \mathrm{mm}\end{array}$ & $\begin{array}{c}\text { Cuadrado Medio } \\
\text { del Error MSE (mm) }\end{array}$ & $\begin{array}{c}\text { Raíz del Cuadrado } \\
\text { Medio del Error RMSE (mm) }\end{array}$ \\
ETo (Estación) & 281 & 1455 & 0,71 & 0,84 \\
ETo (Atmómetro) & 281 & 1495 & & \\
\hline
\end{tabular}

Tabla 2. Resumen de riegos aplicados en el cultivo de frijol.

\begin{tabular}{lcccccc}
\hline Métodos de riego & $\begin{array}{c}\mathrm{N}^{\text {o de }} \\
\text { Riego }\end{array}$ & $\begin{array}{c}\text { Superficie } \\
(\mathrm{ha})\end{array}$ & $\begin{array}{c}\text { Vol. Aplicado } \\
\left(\mathrm{m}^{3}\right)\end{array}$ & $\begin{array}{c}\text { Ln } \\
(\mathrm{cm})\end{array}$ & $\begin{array}{c}\text { Lb } \\
(\mathrm{cm})\end{array}$ & $\begin{array}{c}\text { EA } \\
(\%)\end{array}$ \\
\hline Atmómetro & 16 & 0,86 & 3229 & 33,58 & 37,55 & 89 \\
IrriModel & 17 & 0,86 & 3342 & 35,13 & 38,87 & 90 \\
\hline
\end{tabular}

Ln: Lámina neta, Lb: Lámina bruta, EA: Eficiencia de Aplicación.

experimental de IrriModel. Martínez-Gamiño et al. (2006) observaron rendimiento similar en el cultivo de frijol bajo sistema de riego por goteo desde 2,25 hasta 5,67 th $\mathrm{a}^{-1}$ con diferentes dosis de fertilización.

\section{Productividad del agua}

Se obtuvo la productividad del agua para cada una de las parcelas en función de la cantidad de agua aplicada en $\mathrm{m}^{3}$. En la programación de los riegos con el atmómetro fue de $0,71 \mathrm{~kg} \mathrm{~m}^{-3}$ y $0,68 \mathrm{~kg} \mathrm{~m}^{-3}$ con el IrriModel. Ugalde-Acosta et al. (2011) obtuvieron eficiencia en el uso del agua de $0,68 \mathrm{~kg} \mathrm{~m}^{-3}$ en frijol cultivado bajo riego por goteo.

\section{Conclusiones}

Por su simplicidad de instalación, movilidad, costo accesible para los productores y registro de datos, el atmómetro es la opción viable para estimar la evapotranspiración de referencia, comparada con el costo y mantenimiento de una estación meteorológica automática. Por lo tanto, con una buena operación y mantenimiento, el atmómetro es una alternativa confiable para la calendarización del riego en cultivos con el fin de optimizar este recurso hídrico. Todo lo expuesto sustenta la importancia de implementar el uso de esta tecnología en las zonas agrícolas del país, para el manejo más eficiente y oportuno en la utilización del agua para riego, además de incrementar la eficiencia de aplicación y la productividad del agua. 


\section{Literatura Citada}

Allen, R.G.; Pereira, L.S.; Raes, D. and Smith, M. 1998. Crop evapotranspiration: Guidelines for computing crop water requirements, FAO Irrigation and drainage paper $\mathrm{N}^{\circ} 56$, Food and Agriculture Organization of the United Nations (FAO). Rome, Italy. 15 p.

Bessembinder, J.E.; Leffelaar, P.A.; Dhindwal, A.S.; and

Ponsioen, T.C.

2005. Which crop and which drop, and the scope for improvement of water productivity. Agricultural Water Management, 73(2): 113-130.

Comisión Nacional del Agua

2015. Estadísticas agrícolas de los distritos de riego. Año agrícola 2013/14. CONAGUA. México. 290 p.

Chen, F.; Robinson, P.J.

2009. Estimating reference crop evapotranspiration with

ETgages. Journal of Irrigation and Drainage Engineering,

135(3): 335-342.

Douglas, E.M.; Jacobs, J.M.; Sumner, D.M.; Ray, R.L.

2009. A comparison of models for estimating potential evapotranspiration for Florida land cover types. J. Hydrol., 9(11):366-376.

Domingo, F.; Villagarcía, L.; Brenner, A.J.; and Puidefábregas, J. 1999. Evapotranspiration model for semi-arid shrub-lands tested against data from SE Spain. Agricultural and Forest Meteorology, 95: 67-84.

Fernández, C.; Corá, J.E.; Campos de Araujo, J.A.

2003. Reference evapotranspiration estimation inside greenhouses. Scientia Agricola, 60 (3): 591-594.

Gavilán, P.; Castillo-Llanque, F.

2009. Estimating reference evapotranspiration with atmometer sin a semiarid environment. Agricultural Water Management, 96 (3): 465-472.

García, M.; Raes, D.; Allen, R.; Herbas, C. 2004. Dynamics of reference evapotranspiration in the Bolivian highlands (Altiplano). Agric. For. Meteorol., 125 (1): 67-82.

Gordillo, S.V.M.; Flores, M.H.; Tijerina, C.L.; Arteaga, R.R. 2014. Estimación de la evapotranspiración utilizando un balance de energía e imágenes satelitales. Revista Mexicana de Ciencias Agrícolas, 5(1): 143-155.
Hadi, H.J.; Farah, A.

2018. Evaluating atmometer performance for estimating reference evapotranspiration in ventilated and unventilated greenhouses. Journal of Irrigation and Drainage Engineering, 144 (7): (0418014-1)-(04018014-10).

Knox, J.; Rodríguez-Díaz, J.; Hess, T.

2011. Estimating Evapotranspiration by Using Atmometers for Irrigation Scheduling in a Humid Environment. J. Irrig. Drain Eng., 137 (11): 685-691.

Martínez-Gamiño, M.A.; Jasso-Chaverría, C.; Huerta-Díaz, J. 2006. Fertirriego y labranza de conservación en la producción de frijol. Terra Latinoamericana, 24 (3): 367-374.

Mendoza-Pérez, C.; Sifuentes-Ibarra, E.; Ojeda-Bustamante, W.; Macías-Cervantes, J.

2016. Response of surface-irrigated corn to regulated deficit irrigation. Ingeniería Agrícola y Biosistemas, 8 (1): 29-40.

Melesse, A.M.; Weng, Q.; Thenkabail, P.S.; Senay, G.B.

2007. Remote sensing sensors and applications in environmental resources mapping and modelling. Sensors, 7 (12): 3209-3241.

Ojeda-Bustamante, W.; Sifuentes-Ibarra, E.; Unland-Weiss, H. 2006. Programación integral del riego en maíz en el Norte de Sinaloa. Agrociencia, 40 (1): 13-25.

Organización Meteorológica Mundial.

2012. Manual de aplicación de normas de enseñanza y formación profesional en meteorología e hidrología. Volumen 1-Meteorologia. OMM. Ginebra, Suiza. 33 p.

Ugalde-Acosta, F.J.; Tosquy-Valle, O.H.; López-Salinas, E.; Francisco-Nicolás, $\mathrm{N}$.

2011. Productividad y rentabilidad del cultivo de frijol con fertirriego en Veracruz, México. Agronomía Mesoamericana, 22 (1): 29-36.

Vásquez-Méndez, R., Ventura-Ramos, E. Jr.; Acosta-Gallegos, J.A. 2011. Habilidad de estimación de los métodos de evapotranspiración para una zona semiárida del centro de México. Revista Mexicana de Ciencias Agrícolas, 2 (3): 399-415. 